\title{
Snakes of Bhandara District, Maharashtra, Central India with Notes on Natural History
}

\author{
Rahul V. Deshmukh¹, Sagar A. Deshmukh², Swapnil A. Badhekar ${ }^{3}$, and Roshan Y. Naitame ${ }^{4}$ \\ ${ }^{1}$ H.N. 26, Teacher Colony, Kalmeshwar, Nagpur, Maharashtra-441501, India (rahul30.sake@gmail.com) \\ ${ }^{2}$ Behind Potdar Nursing Home Kalmeshwar, Nagpur, Maharashtra-441501, India (sd.snakefriend@gmail.com) \\ ${ }^{3}$ TiwaskarWadi near BoudhaVihar, Hingana Raipur, Nagpur, Maharashtra-441110, India (swapnilbadhekar86@gmail.com) \\ ${ }^{4}$ Adyal, Pavani, Bhandara, Maharashtra-441903, India (rymaitame750@gmail.com)
}

Photographs by Sagar A. Deshmukh.

$\mathrm{R}$ esearch on reptiles in India dates back more than 200 years, coinciding with the formation of the erstwhile British East India Company. However, central India has largely been neglected as regards herpetological surveys. The District of Bhandara is in the northeastern part of Maharashtra State and lies entirely within the Wainganga Basin. The first appreciable study was carried out by D'Abreu (1928), who conducted a systematic collection of reptiles for the Central Museum in Nagpur, which provided information on the reptiles of Vidharbha Region in general and Bhandara District in particular. Deshmukh et al. (2015) recorded 30 species of snakes from the adjacent Nagpur District. Based on animal rescues and road surveys, we herein document 33 species of snakes in ten families from Bhandara District, Maharashtra $\left(20^{\circ} 40^{\prime} 00^{\prime \prime}-21^{\circ} 35^{\prime} 00^{\prime \prime N}\right.$, 79³0'00"-8006'00"E; Fig. 1).

A team of four persons on two-wheelers at speeds of about $20 \mathrm{~km} / \mathrm{h}$ surveyed seven tehsils (Tumsar, Mohadi, Sakoli, Bhandara, Lakhani, Lakhandur, and Pauni), two each week, and the adjacent Nagazira Wildlife Sanctuary during morning and evening hours on every Saturday and Sunday from May 2013 to July 2016. We also searched roadside leaf litter, cover objects (i.e., logs, bark, rocks), agricultural fields, and along streams and ponds. In addition, we documented snakes rescued from residential areas of Bhandara District. All snakes were captured, identified, photographed, and released. For identification, we relied on criteria listed in Smith (1943), Daniel (2002), and Whitaker and Captain (2004) and comparisons with specimens in the collections of the Central Zone Regional Centre, Zoological Survey of India, Jabalpur, Madhya Pradesh, and the Bombay Natural History Society, Mumbai, Maharashtra. We also recorded secondary information from reliable sources (observations and previous snakerescue records from a conservationist, NGOs from Adyal [Ashik Naitame] and Bhandara [Raju Baghele], and others from Bhandara District). Data are summarized in Tables 1 and 2, and 32 of the 33 species are illustrated in Figs. 2-12.

Miscellaneous natural history observations included: (1) A Barred Wolfsnake (Lycodon striatus) feeding on a Common

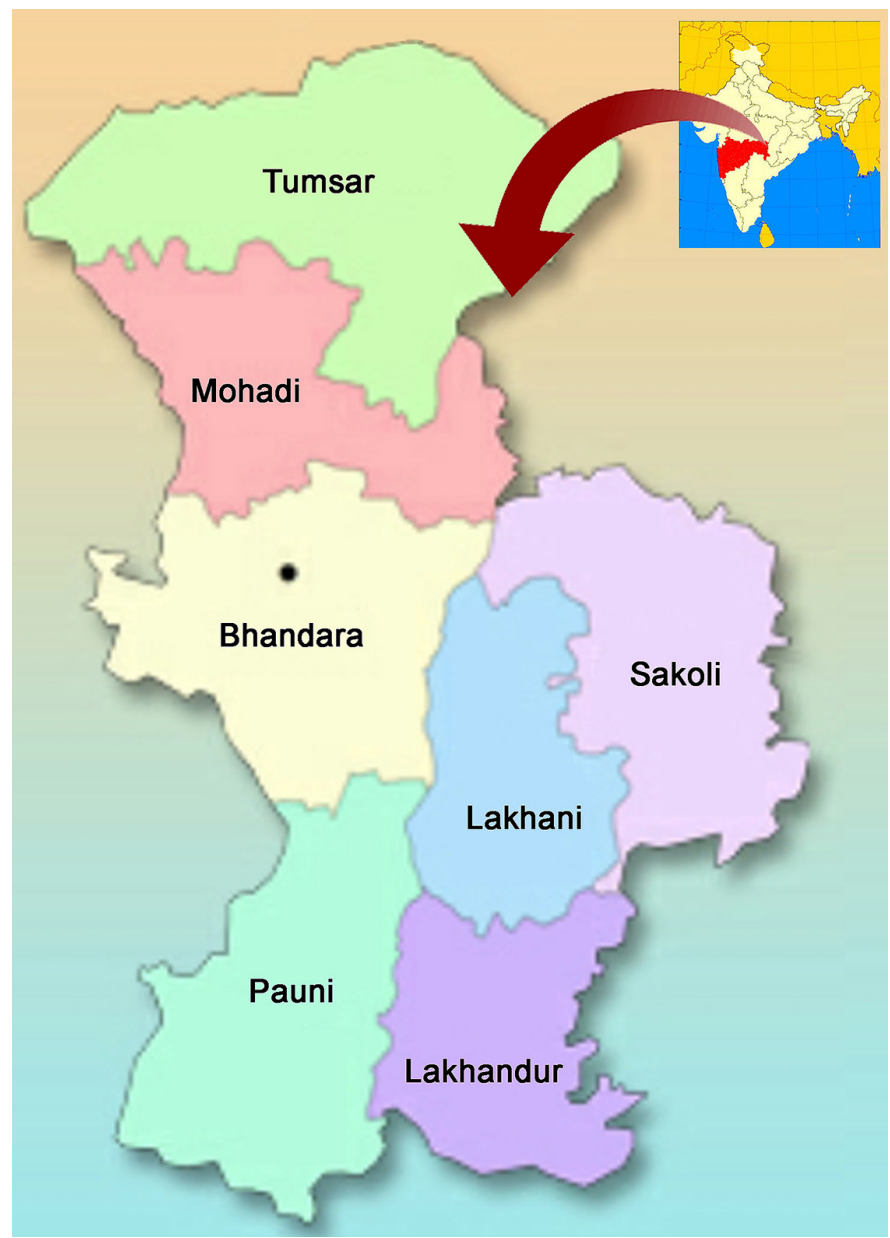

Fig. 1. Location of the study area in Bhandara District, Maharashtra, India. 
House Gecko (Hemidactylus frenatus) in Jawaharnagar, Bhandara City $\left(21^{\circ} 09^{\prime} 11.29^{\prime \prime N}, 79^{\circ} 32^{\prime} 28.70^{\prime \prime E}\right)$ on 26 December 2013; (2) an Oriental Ratsnake (Ptyas mucosa) that had ingested three Checkered Keelbacks (Xenochrophis piscator) on 3 July 2014 along a seasonal stream near Oxygen Park, Samarth Nagar, Lakhani Tehsil $\left(21^{\circ} 04^{\prime} 00.38^{\prime \prime N}\right.$,

Table 1. Snakes encountered during surveys of seven tehsils in Bhandara District, Maharashtra, India.

\begin{tabular}{|c|c|c|c|c|c|}
\hline Tehsil & Dates & Rescued & Roadkilled & Live & Total \\
\hline \multirow[t]{3}{*}{ Tumsar } & May 2013-May 2014 & 74 & 22 & 15 & 111 \\
\hline & June 2014-June 2015 & 126 & 55 & 20 & 201 \\
\hline & July 2015-July 2016 & 70 & 19 & 6 & 95 \\
\hline \multirow[t]{3}{*}{ Mohadi } & May 2013-May 2014 & 85 & 15 & 3 & 103 \\
\hline & June 2014-June 2015 & 103 & 69 & 16 & 188 \\
\hline & July 2015-July 2016 & 47 & 64 & 13 & 124 \\
\hline \multirow[t]{3}{*}{ Sakoli } & May 2013-May 2014 & 40 & 17 & 11 & 68 \\
\hline & June 2014-June 2015 & 100 & 37 & 8 & 145 \\
\hline & July 2015-July 2016 & 64 & 36 & 12 & 112 \\
\hline \multirow[t]{3}{*}{ Bhandara } & May 2013-May 2014 & 59 & 13 & 18 & 90 \\
\hline & June 2014-June 2015 & 173 & 76 & 24 & 273 \\
\hline & July 2015-July 2016 & 35 & 42 & 9 & 86 \\
\hline \multirow[t]{3}{*}{ Lakhani } & May 2013-May 2014 & 53 & 57 & 23 & 133 \\
\hline & June 2014-June 2015 & 99 & 31 & 18 & 148 \\
\hline & July 2015-July 2016 & 87 & 37 & 4 & 128 \\
\hline \multirow[t]{3}{*}{ Lakhandur } & May 2013-May 2014 & 66 & 19 & 11 & 96 \\
\hline & June 2014-June 2015 & 88 & 42 & 9 & 139 \\
\hline & July 2015-July 2016 & 129 & 61 & 13 & 203 \\
\hline \multirow[t]{3}{*}{ Pauni } & May 2013-May 2014 & 125 & 38 & 19 & 182 \\
\hline & June 2014-June 2015 & 82 & 43 & 17 & 142 \\
\hline & July 2015-July 2016 & 66 & 46 & 9 & 121 \\
\hline
\end{tabular}
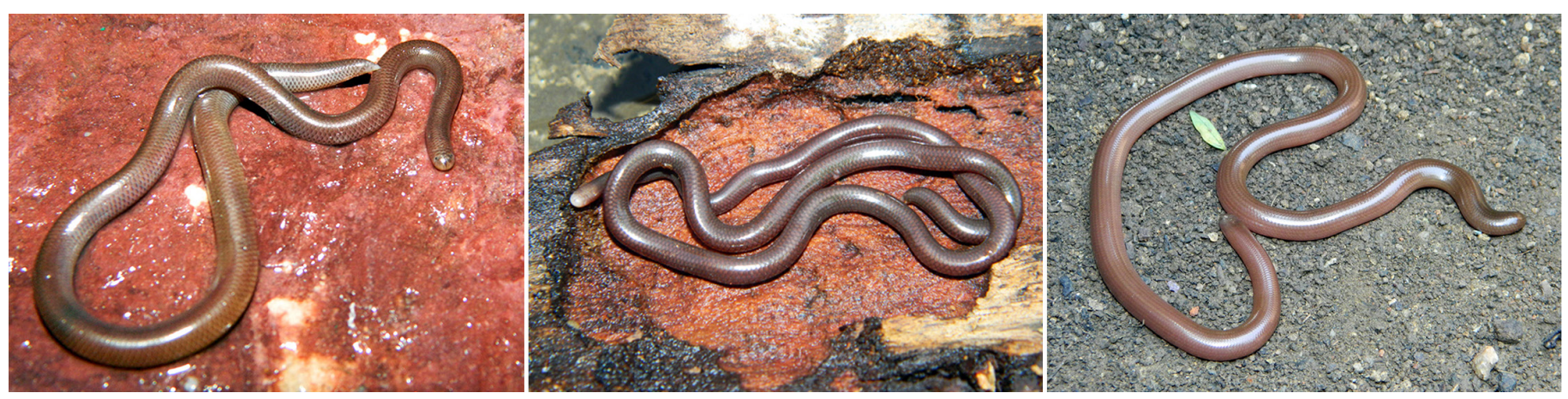

Fig. 2. Brahminy Blindsnake (Indotyphlops braminus) from Mahalgaon Village, Mohadi Tehsil (left). Slender Wormsnake (Indotyphlops porrectus) from Lakhandur Tehsil (center). Beaked Wormsnake (Grypotyphlops acutus) from Hardoli, Mohadi Tehsil (right). 
7949'30.16"E); (3) 22 hatchling Oriental Ratsnakes emerging from eggs on 30 August 2015 in rocky debris at Nirgudi Village in Pavni Tehsil (20²'29.85"N, 7941'01.15"E); (4) a partial albino Common Trinket Snake (Coelognathus helena) roadkilled on the Kosamtondi-Sakoli Road near Ashoka Toll Plaza on 4 September 2015 in Sakoli Tehsil $\left(21^{\circ} 05^{\prime} 17.45^{\prime \prime} \mathrm{N}\right.$, $80^{\circ} 01$ '36.68"E); (5) a young Common Catsnake (Boiga trigonata) feeding on a Common House Gecko (Hemidactylus frenatus) in a garden near Gandhi Sagar Lake, Rajendra Nagar,

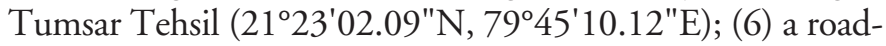
killed female Common Catsnake with two incompletely developed eggs on 14 August 2012 on the GadhegaonChikhalabodhi Road in Lakhani Tehsil $\left(21^{\circ} 04^{\prime} 34.61^{\prime \prime N}\right.$, 79०46'36.98"E); (7) a pair of Striped Keelbacks (Amphiesma stolatum) mating in brick debris $\left(21^{\circ} 03^{\prime} 37.03^{\prime \prime} \mathrm{N}\right.$, $\left.79^{\circ} 51^{\prime} 23.14 " \mathrm{E}\right)$ at Manegaon in Lakhani Tehsil on 21 July 2014; (8) two adult Green Keelbacks (Rhabdophis plumbicolor) attempting to swallow one another near Belgota Village in Pauni Tehsil $\left(20^{\circ} 46^{\prime} 39.95^{\prime \prime} \mathrm{N}, 79^{\circ} 38^{\prime} 21.38^{\prime \prime} \mathrm{E}\right)$ on 5 October 2013; (9) a live Olive Keelback (Atretium schistosum) trapped in a fishing net in a pond at Sawargaon in Lakhandur Tehsil $\left(20^{\circ} 43^{\prime} 35.72 " \mathrm{~N}, 79^{\circ} 52^{\prime} 20.99^{\prime \prime E}\right)$ on 28 February 2015; (10) a Saw-scaled Viper (Echis carinatus) feeding on a frog on 13 September 2014 in Teak leaf litter in the Davezari Village area in Tumsar Tehsil $\left(21^{\circ} 27^{\prime} 02.51^{\prime \prime N}, 79^{\circ} 47^{\prime} 19.20 " \mathrm{E}\right)$; (11) a Slender Coralsnake (Calliophis malanurus) regurgitating a Brahminy Blindsnake (Indotyphlops braminus) in an open sewage line on 21 April 2013 at Paraswada Village in Mohadi Tehsil (2120'26.39"N, 7943'32.07"E); (12) a Common Indian Krait (Bungarus caeruleus) feeding on a Common
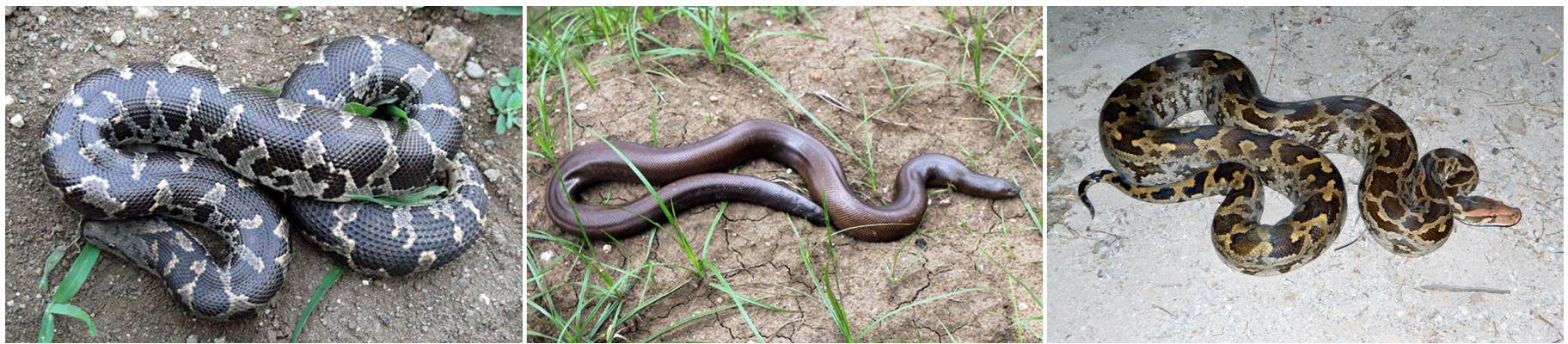

Fig. 3. Common Sand Boa (Eryx conicus) from Manegaon Village, Lakhani Tehsil (left). Red Sand Boa (Eryx johnii) from the Bhandara-Ramtek Road, Tumsar Tehsil (center). Indian Rock Python (Python molurus) from Tamaswadi Village, Tumsar Tehsil (right).
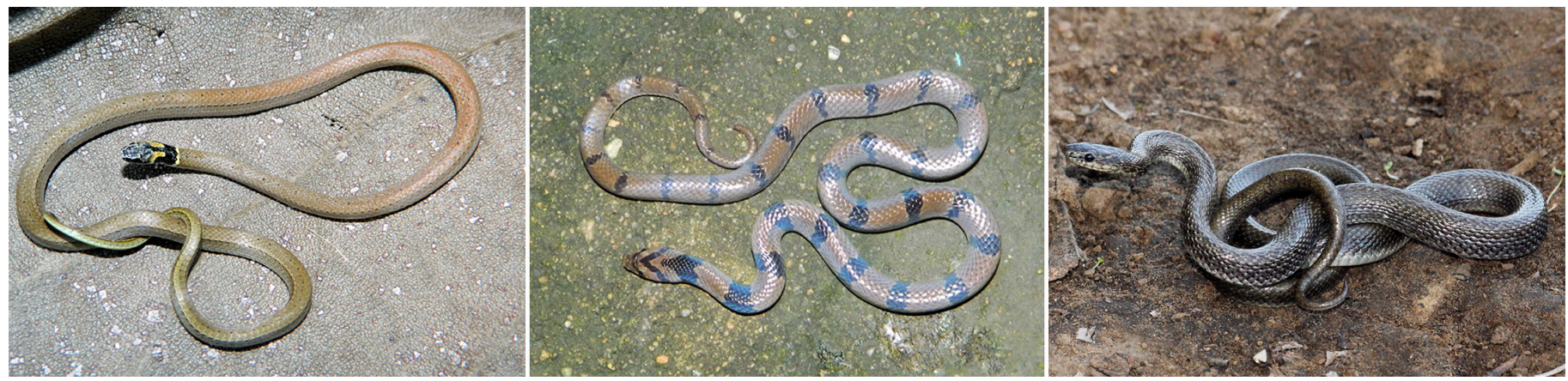

Fig. 4. Duméril's Black-headed Snake (Sibynophis subpunctatus) from Tumsar Tehsil (left). Common Kukri Snake (Oligodon arnensis) from Nilaj, Sakoli Tehsil (center). Indian Smooth Snake (Coronella [= Wallophis] brachyura) from the outskirts of the Gaidongari Reserve Forest, Pauni Tehsil (right).
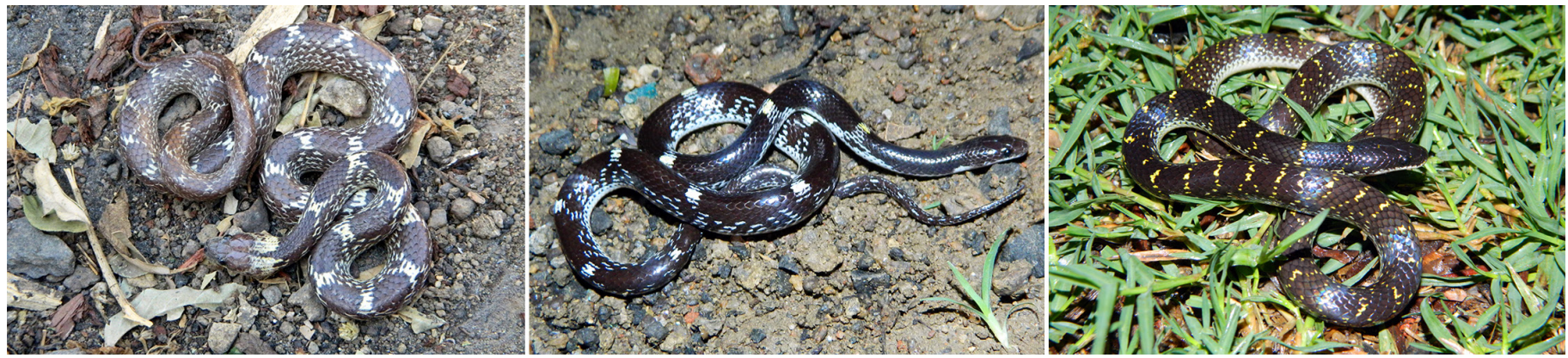

Fig. 5. Common Wolfsnake (Lycodon aulicus) from Mohadi Tehsil (left). Barred Wolfsnake (Lycodon striatus) from Jawahar Nagar, Bhandara City (center). Yellow-spotted Wolfsnake (Lycodon flavomaculatus) from the outskirts of Chinchgaon Village Lakhandur Tehsil (right). 

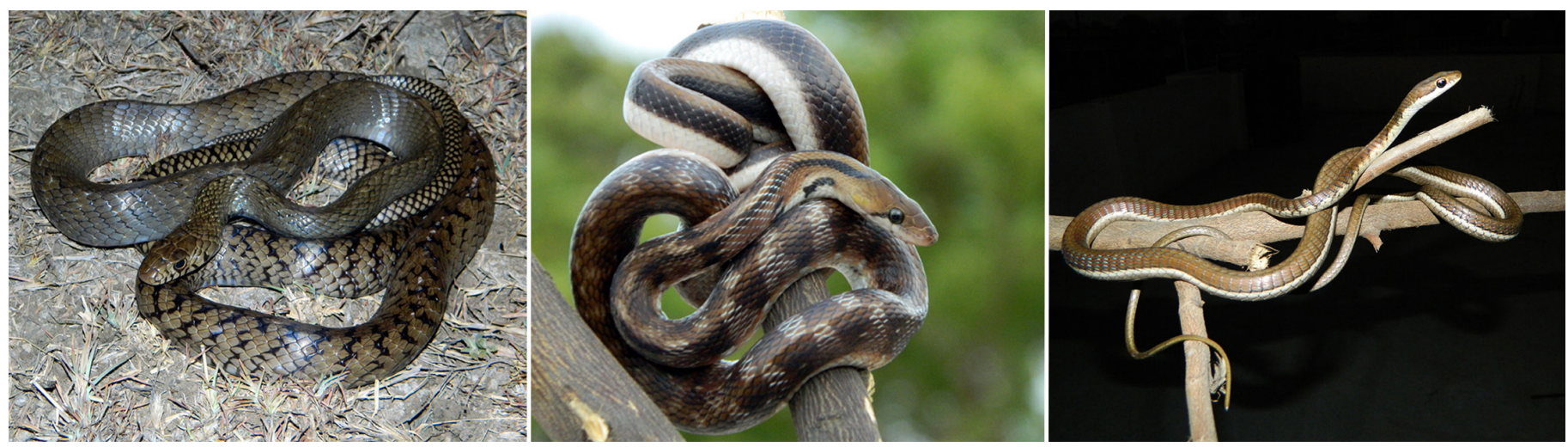

Fig. 6. Oriental Ratsnake (Ptyas mucosa) from Tumsar Tehsil (left). Common Trinket Snake (Coelognathus helena) from Sakoli Tehsil (center). Common Bronze-backed Treesnake (Dendrelaphis tristis) from Pauni Tehsil (right).
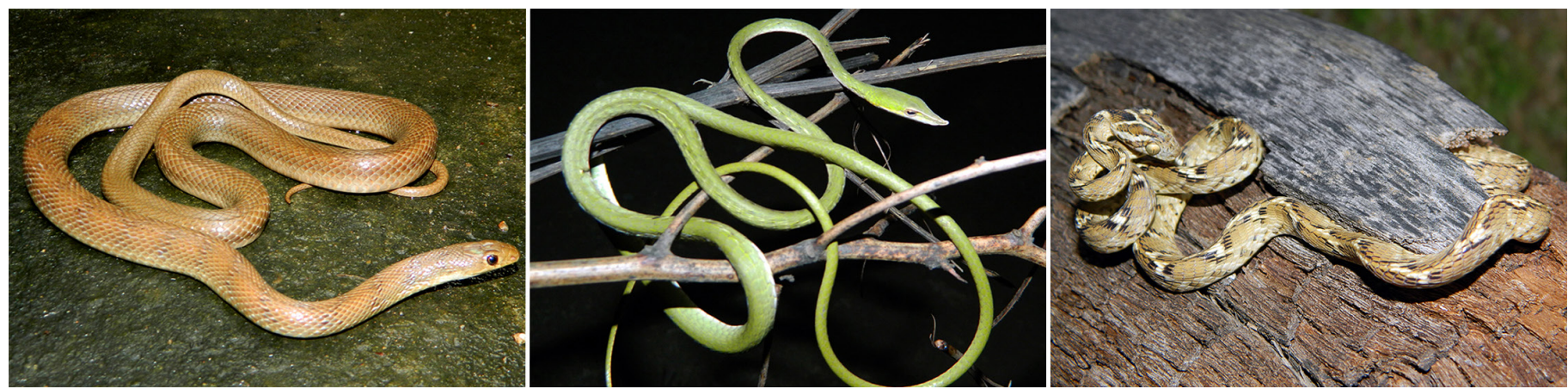

Fig. 7. Banded Racer (Argyrogena fasciolata) from Ganeshpur Khairy, Tumsar Tehsil (left). Long-nosed Whipsnake (Ahaetulla nasuta) from Pauni Tehsil (center). Common Catsnake (Boiga trigonata) from Chinchkhed, Mohadi Tehsil (right).
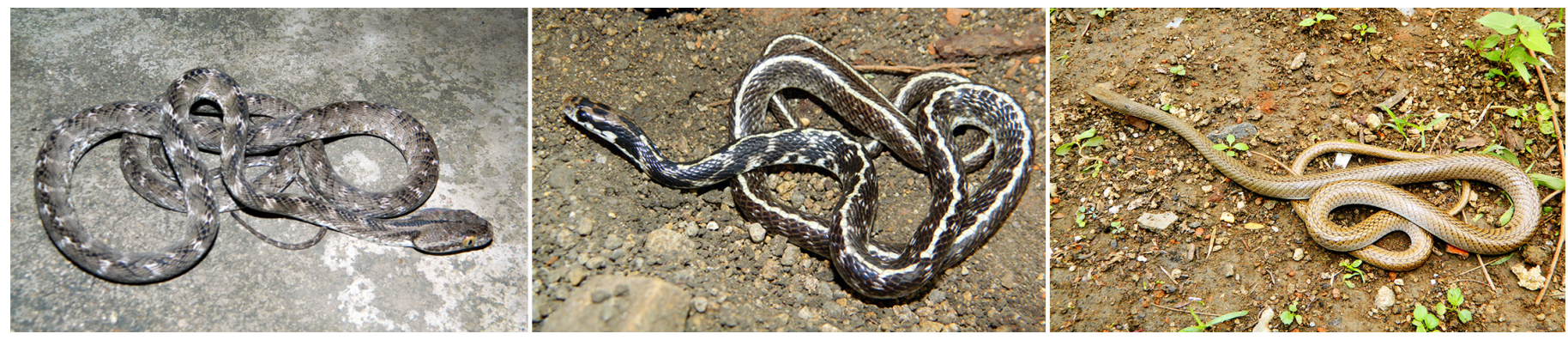

Fig. 8. Forsten's Catsnake (Boiga forsteni) from the temple in Adyal, Pauni Tehsil (left). Indian Egg-eater (Elachistodon [= Boiga] westermanni) from Pauni Tehsil (center). Stout Sandsnake (Psammophis longifrons) from Pauni Tehsil (right).

Wolfsnake (Lycodon aulicus) during a rescue operation in the Hanuman Temple near Khairlanji Village $\left(21^{\circ} 22^{\prime} 05.13^{\prime \prime N}\right.$, 79'34'26.60"E) on 19 November 2015; (13) an adult male Indian Cobra (Naja naja) regurgitating a Common Sand Boa (Eryx conicus) in an agricultural field near Lakhani (210.'1.03"N, 7949'37.42"E).

Nande and Deshmukh (2007) recorded the Montane Trinket Snake (Coelognathus helena monticollaris) and Wall's Krait (Bungarus walli) in Amravati District (which is adjacent to Bhandara District). We found neither during our surveys, although we did find Common Trinket Snakes $(C$. helena helena). We also failed to find Travancore Wolfsnakes (Lycodon travancoricus), Slender Racers (Platyceps gracilis), or Calamaria Reedsnakes (Liopeltis calamaria), all of which had been collected by D'Abreu (1928) from the Central Provinces (now Madhya Pradesh, Chhattisghar, and Vidharbha). However, the Slender Wormsnake (Indotyphlop sporrectus), Dumeril's Black-headed Snake (Sibynophis subpunctatus), Streaked Kukri Snake (Oligodon taeniolatus), Indian Smooth Snake (Coronella [= Wallophis] brachyura), Barred Wolfsnake (Lycodon striatus), Yellow-Spotted Wolfsnake (Lycodon flavomaculatus), Olive Keelback (Atretium schistosum), Forsten's Catsnake (Boiga forsteni), Indian Eggeater (Elachistodon $[=$ Boiga $]$ westermanni), Stout Sandsnake (Psammophis longifrones), Bamboo Pitviper (Trimeresurus gramin- 
Table 2. Snakes encountered during surveys of seven tehsils in Bhandara District, Maharashtra, India. Abundance: $\mathrm{R}=\mathrm{Rare}$, UC = Uncommon, $\mathrm{C}=$ Common, $\mathrm{VC}=$ Very Common. Habitat: $\mathrm{SL}=$ scrubland, $\mathrm{PD}=$ ponds, $\mathrm{SS}=$ streams, $\mathrm{AF}=$ agricultural fields, $\mathrm{RD}=$ rocks, LF = leaf litter. IUCN Red List status: NE = Not Evaluated, LC = Least Concern, NT = Near Threatened.

$\begin{array}{lllll}\text { Species } & \text { Number } & \text { Abundance } & \text { Habitat } & \text { IUCN Status }\end{array}$

\section{TYPHLOPIDAE}

Brahminy Blindsnake

35

C

LF, RD, AF, PD

NE

(Indotyphlops braminus)

Slender Wormsnake

17

C

LF, RD, AF, PD

NE

(Indotyphlops porrectus)

Beaked Wormsnake

27

C

LF, RD, AF, PD

$\mathrm{NE}$

(Grypotyphlops acutus)

\section{ERYCIDAE}

Common Sand Boa 37

C

AF, PD, SS

NE

(Eryx conicus)

Red Sand Boa 7

UC

AF, SL

NT

(Eryx johnii)

\section{PYTHONIDAE}

Indian Rock Python

9

UC

PD, SS, AF, SL

NT

(Python molurus)

\section{SIBYNOPHIIDAE}

Duméril's Black-headed Snake

13

UC

LF, RD, AF

NE

(Sibynophis subpunctatus)

\section{COLUBRIDAE}

Common Kukri Snake

C

SL, PD, SS, AF, RD, LF

$\mathrm{NE}$

(Oligodon arnensis)

Streaked Kukri Snake

(Oligodon taeniolatus)

Indian Smooth Snake

6

UC

AF

NE

(Coronella [= Wallophis $]$ brachyura)

Common Wolfsnake

5

$5 \quad \mathrm{R}$

$\mathrm{R} \quad \mathrm{AF}$

$\mathrm{NE}$

(Lycodon aulicus)

Barred Wolfsnake 379

VC

SL, AF, RD

$\mathrm{NE}$

(Lycodon striatus) 14

UC

AF

NE

Yellow-spotted Wolfsnake

13

UC

AF, SL

LC

(Lycodon flavomaculatus)

Oriental Ratsnake 390

VC

AF, RD, SL, PD, SS, LF

NE

(Ptyas mucosa)

Common Trinket Snake

189

C

AF, RD, PD, SS, LF, SL

$\mathrm{NE}$

(Coelognathus helena)

Banded Racer

100

C

RD, AF, SL

NE

(Argyrogena fasciolata) 
(continued from previous page)

\begin{tabular}{|c|c|c|c|c|}
\hline Species & Number & Abundance & Habitat & IUCN Status \\
\hline Common Catsnake & 39 & $\mathrm{C}$ & SL, AF & $\mathrm{NE}$ \\
\hline \multicolumn{5}{|l|}{ (Boiga trigonata) } \\
\hline Forsten's Catsnake & 13 & UC & SL & $\mathrm{NE}$ \\
\hline \multicolumn{5}{|l|}{ (Boiga forsteni) } \\
\hline Indian Egg-eater & 4 & $\mathrm{R}$ & SF, AF, LF & LC \\
\hline \multicolumn{5}{|l|}{ (Elachistodon $[=$ Boiga $]$ westermanni) } \\
\hline \multicolumn{5}{|l|}{ AHAETULIIDAE } \\
\hline Long-nosed Whipsnake & 8 & UC & SL & $\mathrm{NE}$ \\
\hline \multicolumn{5}{|l|}{ (Ahaetulla nasuta) } \\
\hline Common Bronze-backed Treesnake & 45 & $\mathrm{C}$ & SL, AF & $\mathrm{NE}$ \\
\hline \multicolumn{5}{|l|}{ (Dendrelaphis tristis) } \\
\hline \multicolumn{5}{|l|}{ PSAMMOPHIIDAE } \\
\hline Stout Sandsnake & 16 & UC & $\mathrm{SL}, \mathrm{AF}, \mathrm{RD}, \mathrm{LF}$ & $\mathrm{LC}$ \\
\hline \multicolumn{5}{|l|}{ (Psammophis longifrons) } \\
\hline \multicolumn{5}{|l|}{ NATRICIDAE } \\
\hline Striped Keelback & 66 & $\mathrm{C}$ & AF, SL, SS, LF, RD & $\mathrm{NE}$ \\
\hline \multicolumn{5}{|l|}{ (Amphiesma stolatum) } \\
\hline Green Keelback & 36 & $\mathrm{C}$ & $\mathrm{AF}, \mathrm{RD}, \mathrm{SL}$ & $\mathrm{NE}$ \\
\hline \multicolumn{5}{|c|}{ (Macropisthodon $[=$ Rhabdophis $]$ plumbicolor) } \\
\hline Checkered Keelback & 609 & $\mathrm{VC}$ & $\mathrm{PD}, \mathrm{SS}, \mathrm{AF}$ & $\mathrm{NE}$ \\
\hline \multicolumn{5}{|l|}{ (Fowlea $[=$ Xenochrophis $]$ piscator) } \\
\hline Olive Keelback & 13 & UC & ST, PD, AF & LC \\
\hline
\end{tabular}

(Atretium schistosum)

\section{VIPERIDAE}

\begin{tabular}{|c|c|c|c|c|}
\hline Russell's Viper & 250 & $\mathrm{C}$ & SL, AF, LF, RD & $\mathrm{NE}$ \\
\hline \multicolumn{5}{|l|}{ (Daboia russelii) } \\
\hline Saw-scaled Viper & 7 & $\mathrm{R}$ & SL, LF, RD & $\mathrm{NE}$ \\
\hline \multicolumn{5}{|l|}{ (Echis carinatus) } \\
\hline Bamboo Pitviper & 1 & $\mathrm{R}$ & SL, SS & LC \\
\hline \multicolumn{5}{|l|}{ (Trimeresurus gramineus) } \\
\hline \multicolumn{5}{|l|}{ ELAPIDAE } \\
\hline Slender Coralsnake & 6 & UC & $\mathrm{LF}, \mathrm{AF}, \mathrm{RD}, \mathrm{SL}$ & $\mathrm{NE}$ \\
\hline \multicolumn{5}{|l|}{ (Calliophis melanurus) } \\
\hline Common Indian Krait & 79 & $\mathrm{C}$ & RD, LF, SL, AF & $\mathrm{NE}$ \\
\hline \multicolumn{5}{|l|}{ (Bungarus caeruleus) } \\
\hline Banded Krait & 41 & $\mathrm{C}$ & $\mathrm{SS}, \mathrm{PD}, \mathrm{LF}, \mathrm{AF}$ & LC \\
\hline \multicolumn{5}{|l|}{ (Bungarus fasciatus) } \\
\hline Indian Cobra & 345 & $\mathrm{C}$ & AF, SS, PD, RD, LF, SL & $\mathrm{NE}$ \\
\hline (Naja naja) & & & & \\
\hline
\end{tabular}



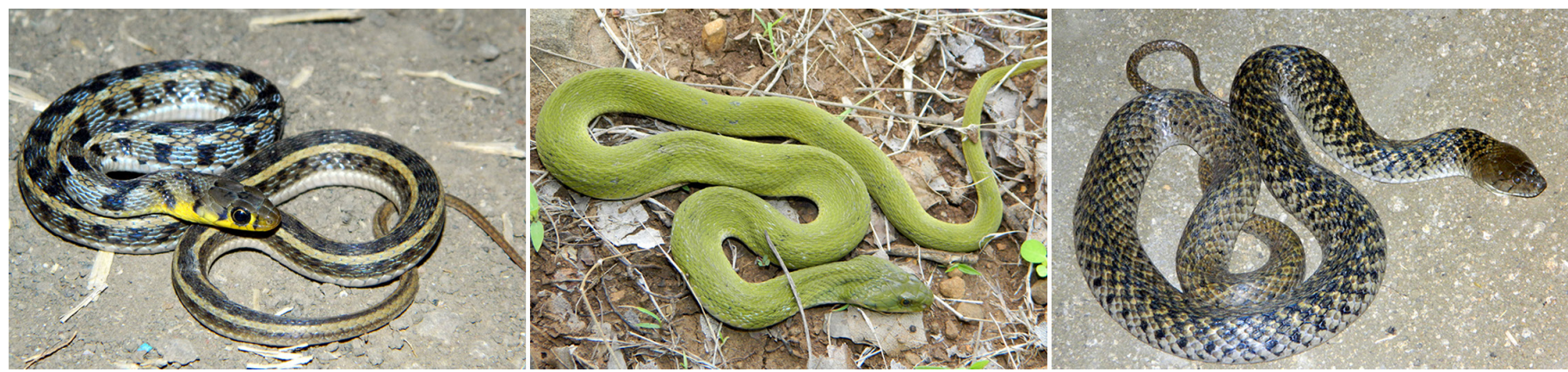

Fig. 9. Striped Keelback (Amphiesma stolatum) from Lakhandur Tehsil (left). Green Keelback (Macropisthodon [= Rhabdophis] plumbicolor) from Lakhani Tehsil (center). Checkered Keelback (Fowlea [= Xenochrophis] piscator) from Mohadi Tehsil (right).
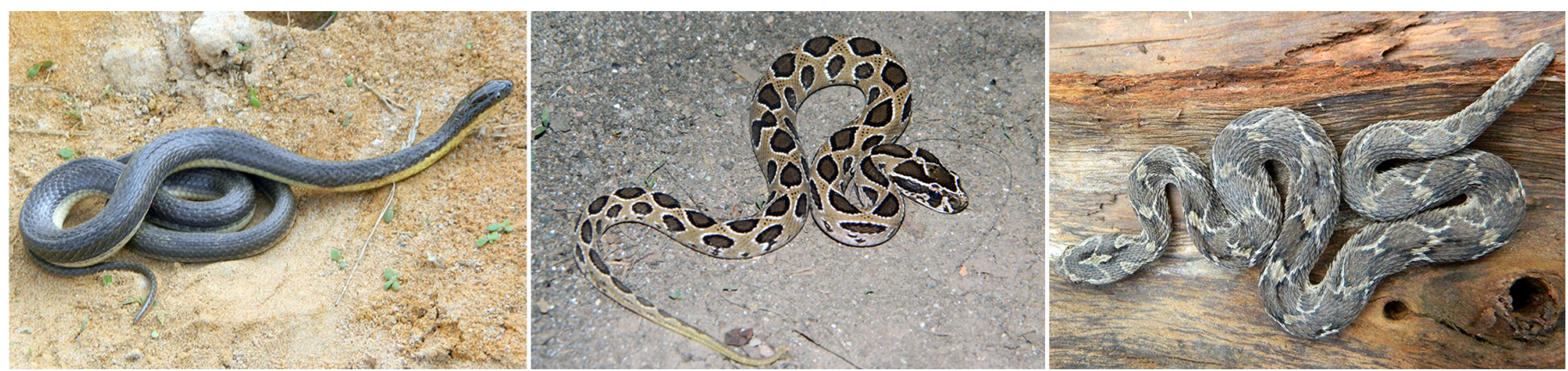

Fig. 10. Olive Keelback (Atretium schistosum) from the Bhandara Road in Pauni Tehsil (left). Russell's Viper (Daboia russelii) from Sakoli Tehsil (center). Saw-scaled Viper (Echis carinatus) from Tumsar Tehsil (right).
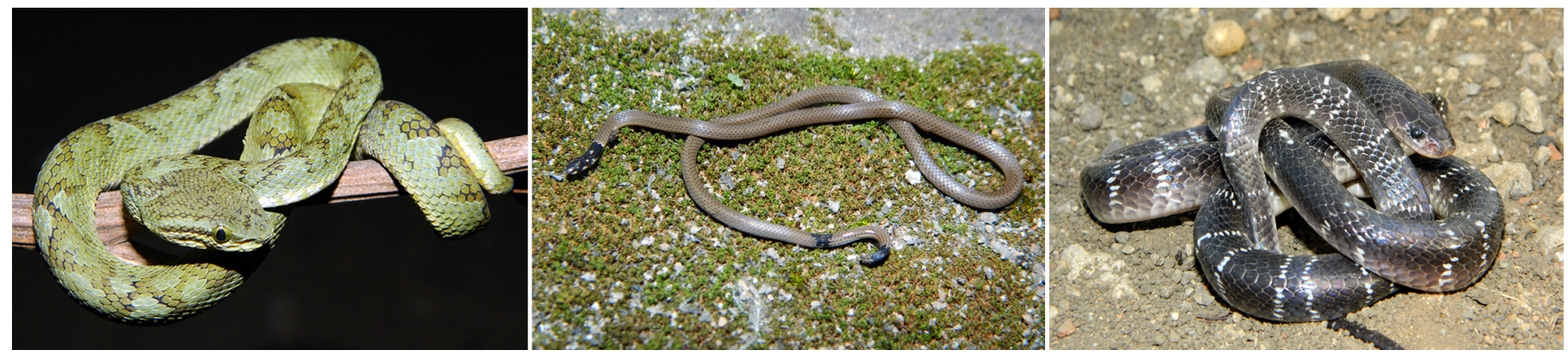

Fig. 11. Bamboo Pitviper (Trimeresurus gramineus) from Pauni Tehsil (left). Slender Coralsnake (Calliophis melanurus) from Mohadi Tehsil (center). Common Indian Krait (Bungarus caeruleus) from Lakhandur Tehsil (right).

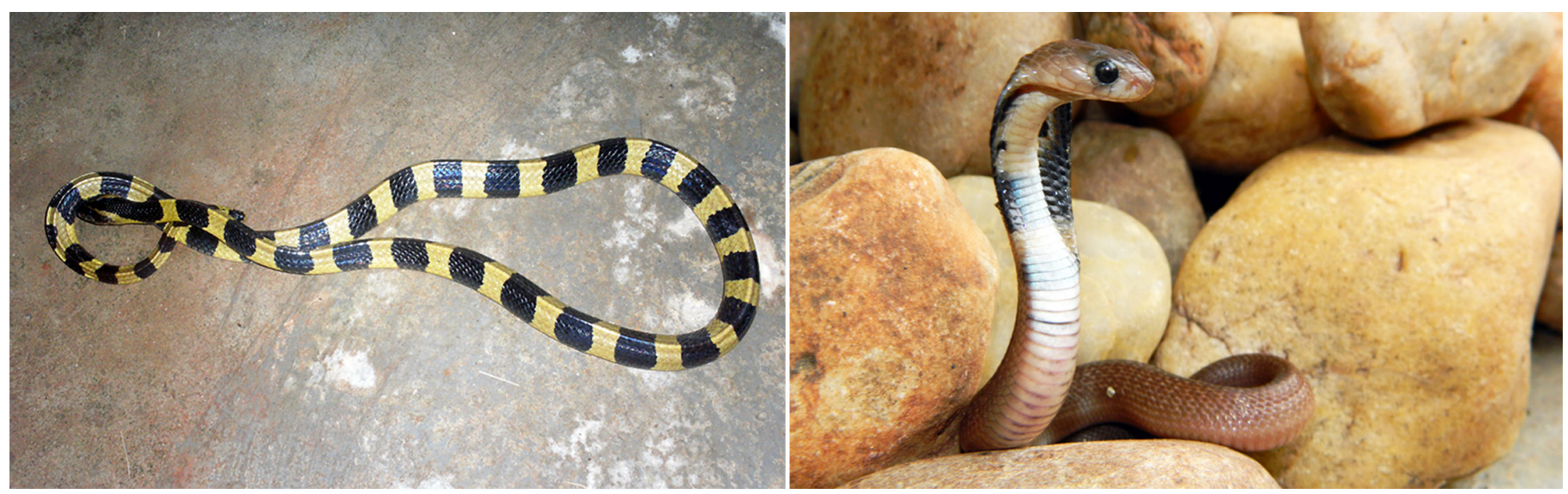

Fig. 12. Banded Krait (Bungarus fasciatus) from Pauni Tehsil (left). Indian Cobra (Naja naja) from Pauni Tehsil (right). 
sis), and Slender Coralsnake (Calliophis malanurus) are recorded herein for the first time in Bhandara District.

\section{Acknowledgements}

Our sincere thanks to Ashik Naitame, Raj Baghele, Ajay Pachare, Pankaj Bhivgade, Sandeep Shende, and Shubham Katgube.

\section{Literature Cited}

D'Abreu, A.E. 1928. Records of the Nagpur Museum No. VI. A List of the Reptiles of the Central Provinces. Government Press, Nagpur, India.
Deshmukh, R.V., S.A. Deshmukh, and S.A. Badhekar. 2015. Rescued records of snakes from Nagpur District, Maharashtra with data on unrecorded species. Reptile Rap 17: 34-43.

Daniel, J.C. 2002. The Book of Indian Reptiles and Amphibians. Bombay Natural History Society and Oxford University Press, Mumbai, India.

Nande, R. and S. Deshmukh. 2007. Snakes of Amravati District including Melghat, Maharashtra, with important records of Indian Egg-Eater, Montane Trinket Snake and Indian Smooth Snake. Zoos' Print Journal 22: 2920-2924.

Smith, M.A. 1943. The Fauna of British India, Ceylon and Burma, Including the Whole of the Indo-Chinese Sub-region. Reptilia and Amphibia. Vol. III.Serpentes. Taylor and Francis, London, UK.

Whitaker, R. and A. Captain. 2004. Snakes of India. The Field Guide. Draco Books, Chennai, India. 